\title{
Zur Kommunikationsausbildung in Asien: Eine Überlegung nach zehn Jahren
}

\author{
von John Mitchell
}

Dr. Jürgen Ruesch, ein Psychiater mit umfassendem Arbeitsgebiet, stellte sich vor einigen Jahren die Aufgabe, eine Liste der gängigen Arbeitsbereiche der Kommunikation zusammenzustellen. Diese Liste wies das jeweilige Spezialgebiet aus und nannte einige maßgebende Quellen, nach denen jedem dieser Árbeitsbereiche eine größere Bedeutung für das Kommunikationsstudium zugeschrieben wurde. Seine Liste umfaßte Gebiete aus der Mathematik und Ingenieurwissenschaft bis zu Innenarchitektur und Zimmerspielen. Sie erstreckte sich auf Psychophysiologie, Neurophysiologie und Psychopharmakologie. Insgesamt führte sie 40 verschiedene Methoden des Kommunikationsumgangs auf. Sie wies flüchtig auf sexuelle Beziehungen als eine Spielart von Kommunikation hin; bei den schönen Künsten, dem Kunsthandwerk und den Gesellschaftsspielen brauchte sie lediglich zehn neue Kategorien einzuführen. Theoretisch ließen sich noch fünf Kategorien hinzufügen. Das bedeutet, daß es zur Zeit 55 verschiedene wissenschaftliche Zugänge zur Kommunikation gibt. Die Schätzung von 40 Methoden ist gewiß schon veraltet. Daraus wird deutlich, wie schwierig es ist, zu versuchen, einen Ausbildungsplan für Kommunikation zu erstellen, weil alles, was Menschen tun, in irgendeinem Sinn unter das Generalthema „Kommunikation“ fällt. Dr. George N. Gordon, ein amerikanischer Kommunikationswissenschaftler, versucht mit einigem Erfolg, wie ich glaube, die Bäume im Wald zu finden.

Erkenntnistheoretische Überlegungen müssen ins Spiel gebracht werden. Ein Voranschreiten im Wald ohne die Auseinandersetzung mit den philosophischen Voraussetzungen führt dazu, daß man die Bäume nicht sieht. Cees Hamelink hat in seinem Buch "Perspectives for Public Communication" gezeigt, wie christliche Kommunikatoren in diesen Fehler verfallen sind. Bestimmte Abschnitte der folgenden Untersuchung werden sich auf die theoretische Grundlage (the "what") der sprachlichen Kommunikation beziehen, Sprachstudium, Linguistik, Semantik, Symbolik und ähnliches einbegriffen. Der am meisten verwirrende Aspekt dieser Theorie-Studie zielt aber auf ein neues Verständnis der sogenannten „Medien“. Wahrscheinlich sind weniger "Medien" am Kommunikationsprozeß beteiligt, als wir früher angenommen haben. Ferner wird es zur Klärung der Zielsetzung beitragen, wenn man zwischen einem Kommunikationsmedium (medium of communication) und einem Kommunikationsinstrument (instrument or device of communication) unterscheidet.

Im Anschluß an die Theorie-Studie umfaßt die Praxis-Studie („how-they-work“ study) andere Gesichtspunkte der Untersuchung. Hier kommen die kognitiven, stilistischen, emotionalen und intentionalen Faktoren ins Spiel. Diese Faktoren stellen Kommunikation manchmal auf eine Stufe mit Kunst. Die Theorie-Studie wird in der

John Mitchell M. A. war bis 1973 Kommunikationsabteilungsleiter der Sogang Universität in Seoul und 1974 bis 1976 in der gleichen Funktion an der "Chinese University“ in Hongkong. 
Anwendung einer wissenschaftlichen Methode im allgemeinen Sinn von Wissenschaft und die Praxis-Studie in einer mehr menschlich intuitiven Methode bestehen, ohne daß beide sich gegenseitig ausschließen. Tatsächlich tendieren beide Methoden auf dem Höhepunkt kreativer Einsicht zur Verschmelzung.

Schließlich müßten, um über die allgemeineren Aspekte des Studiums zu sprechen, die Kommunikationstechnologien untersucht werden. Weil die Technologien schon an der Oberfläche leicht zu erfassen und zu beobachten sind, haben sie unsere äußere Aufmerksamkeit in der Vergangenheit zu sehr gefesselt. Wir waren so fasziniert von diesen neuen Möglichkeiten wie unsere Vorfahren von Tempeln und Kathedralen. Im modernen Asien werden die Antennen und ausgeklügelten Kommunikationssysteme geradezu als Wahrzeichen der Entwicklung verehrt. Die Asiaten stellen sie für fast alle Besucher aus dem Westen zur Schau, um zu zeigen, wie wirkungsvoll die Technologie genutzt wird und wie schnell der Osten gegenüber dem Westen aufholt. Technologien sind in diesen Ländern zu leicht zu beschaffen, und die äußere Faszination überdeckt den tiefgreifenden kulturellen Wandel, den sie mit sich bringen.

Kulturelle Veränderungen und Verwicklungen, die von den Kommunikationstechnologien im Namen einer Entwicklung eingeleitet werden, die in Asien nahezu ganz mit ökonomischer Entwicklung im industriellen Bereich identifiziert wird, sieht man weder voraus noch fürchtet man sie. Die in der Vergangenheit fest gegründeten kulturellen Errungenschaften im Bereich menschlicher Beziehungen, der Familiengemeinschaft, der Kunst, der Religion und der Philosophie, werden beispielsweise von der jüngeren Generation der Asiaten entweder ignoriert oder verachtet. Für sie ist es angemessener, Benson \& Hedges zu rauchen, Levis zu tragen und MacDonald-Hamburger zu essen, als sich mit dem reichen Erbe ihrer Vergangenheit zu identifizieren. Die unglücklichen Folgen dieser Einstellung durch Identitätsverlust und Entfremdung sind in einem schnell entwickelten asiatischen Land wie Japan schon absehbar. Kurz, die kulturellen und interkulturellen Aspekte der Kommunikation müssen in künftigen Ausbildungsprogrammen ein größeres Gewicht erhalten, als das bisher der Fall war.

Ich wende mich in meiner Darstellung nun einer mehr persönlichen Ebene zu. Ihre stark personalistische und individualistische Betrachtungsweise macht sie, so denke ich, nicht weniger allgemeingültig und universal für die Lösung gegenwärtiger und künftiger Ausbildungsprobleme. Ich kam 1966 nach Seoul, Korea, getragen von einer Welle des Enthusiasmus für die Entwicklung der dortigen Massenkommunikation. Von 1958 bis 1961 war ich an der Gründung der Sogang-Universität beteiligt, lernte die koreanische Sprache und erlebte unmittelbar die koreanische Kultur. Mit diesen Erfahrungen kehrte ich wieder in die USA zurück, um weiter zu studieren und eine Strategie für den Bereich der Massenkommunikation an der Sogang-Universität zu erarbeiten. Als ich 1966 zurückkehrte, waren an anderen Universitäten schon Fachbereiche für Journalismus und Rundfunkwesen eingerichtet. Das Schwergewicht im Curriculum dieser Institute lag im Pressewesen. Es gab außerdem Fachbereiche für Theaterwissenschaft (drama), und ein kleines College konzentrierte sich auf Film und Fotografie. Letzteres hatte aber wenig Ansehen und konnte nur mit Mühe qualifizierte Studenten anziehen. Diese Feststellung kann in gewissem Sinne auch für alle jene Fachbereiche gelten, die sich mehr allgemein mit Massenkommunikation befassen. Ich sah deshalb zwei unmittelbare Aufgaben: 1. ein Curriculum aufzustellen, das den neuen und 
wachsenden Bedürfnissen nach Massenkommunikation entsprach, und 2. ein negatives Image zu beseitigen, das die Studenten von diesen Fachbereichen hatten.

Nach Befragung von Medienfachleuten in Korea und anderswo, nach Diskussionen mit anderen akademischen Lehrern und nach dem Studium der jeweiligen Lehrpläne während eines ganzen Jahres stellte ich einen Fachbereichsplan auf. Nach einigen Schwierigkeiten genehmigte das Erziehungsministerium den Fachbereich, und die ersten Studenten trafen im März 1968 ein. Obwohl ich es damals nicht bemerkte, muß ich im Rückblick zugeben, daß es sich eigentlich um eine Abteilung für Kommunikation im amerikanischen Sinne handelte. Ich kam frisch von einer amerikanischen Universität und konnte deswegen nicht an einen eigenständigen koreanischen Fachbereich denken. Fast alle meine Bücher waren amerikanischen Ursprungs. Alle meine Fachkollegen waren in Amerika ausgebildet und hatten dort ihre akademischen Grade erhalten. Dasselbe könnte man allerdings für fast alle vergleichbaren Institutionen Koreas in jener Zeit sagen. Nur wenige Gelehrte waren in Deutschland, Frankreich und Japan ausgebildet worden. Sicherlich richtete sich unser Fachbereich mehr auf amerikanische Institutionen aus als auf andere. Dies läßt sich auch durch die dem Koreakrieg folgende amerikanische Präsenz in Korea erklären, die sich auch auf viele andere Bereiche des koreanischen Lebens auswirkte. Alle Regierungsprogramme für die ökonomische Entwicklung waren z. B. derart beeinflußt. Schließlich bringt eine finanzielle Unterstützung gewollt oder ungewollt größeren Einfluß der Geberländer mit sich. Im Falle Korea ging die Hauptunterstützung von Amerika aus. Meine eigene Universität wurde im wesentlichen mit amerikanischen und westdeutschen Geldern unterstützt.

Damals im März 1968 war der Fachbereich für Massenkommunikation für Lehrer und Studenten eine neue, reizvolle Aufgabe. Die Universität stellte sich durch dieses Fachgebiet in eine Reihe mit den besten Hochschulen. Dies deutete zumindest auf Modernisierung hin. Und wir bewegten uns im Gleichschritt mit dem symbolischen Vormarsch, der den zweiten Fünfjahresplan Koreas vorantrieb. Im wesentlichen konzentrierten wir uns auf "hardware" (hier: Produktionsmittel) mit den Schwerpunkten Rundfunkwesen (Radio und Fernsehen), Theater und Film, weil Korea zu dieser Zeit, ähnlich den neuen Fabriken, gerade diese Fachleute in den Massenmedien benötigte. Es wäre zu viel verlangt gewesen, nicht auf dieser phantastischen Welle von Fortschritt und Enthusiasmus zu schwimmen, sondern gegen den Strom. Auch andere Universitäten erhielten damals "hardware“, und wir wetteiferten miteinander um Schlagzeilen und Fotos in den Zeitungen. Wir waren zu Madison-Avenue-Experten in Anpreisung und Verkauf unserer neuen Arbeitsweise geworden.

Weil in ganz Asien die meisten der armen Nationen dem Mythos der Entwicklung ausgeliefert sind, lassen sich dort ähnliche Phänomene im Bereich der Massenkommunikation beobachten. Die UNESCO rief Projekte ins Leben, und wenn sie nicht direkt Programme aufnahm, die aus der Perspektive Wilbur Schramms von der Stanford Universität gestaltet waren, verbreitete sie zumindest den Mythos und die Fixierung bzw. die Faszination auf "hardware“. Demnach war "hardware " für technologisch ausgefeilte Ubermittlungsprozesse ebenso Teil der modernen Industrielandschaft wie Fabriken und Industriegebiete. Industrialisierung und Modernisierung werden von den reichen Nationen als ein fertiges Paket übergeben. Die armen Nationen dürfen nicht auswählen. Mit der Verpackung des Geschenks beschäftigt, kümmern sie sich wenig um den Inhalt - wenigstens zu Anfang. Aber der anfängliche Fehler, nur die Verpackung sorgfältig zu untersuchen, wird in den asiatischen Ländern jetzt registriert. 
Einige der internationalen Organisationen, die bei der rapiden Entwicklung von "hardware" für Massenkommunikation geholfen haben, beispielsweise UNESCO, WACC, UNDA und AMIC, sehen ein, daß mit Sendemasten, Satelliten für Erziehungsprogramme, wirkungsvollen Produktionszentren u. ä. eine Entwicklung nach den wirklichen Bedingungen und Bedürfnissen asiatischer Länder in weite Ferne gerüdkt und als Resultat eine ausländische kulturideologische Herrschaft errichtet wird. Für den äußerst einseitigen globalen Informationsfluß folgert Tapio Varis: „Man kann annehmen, daß der gesamte Kommunikationsfluß, der zwischen den industrialisierten Teilen der Welt, die von etwa einem Drittel der Menschheit bewohnt werden, und der Dritten Welt (etwa zwei Drittel umfassend) stattfindet, ungefähr einhundert Mal mehr in Richtung von den industrialisierten Ländern zu den Entwicklungsländern verläuft als umgekehrt." Die besondere amerikanische Vorherrschaft in den Rundfunk-Medien ist von Herbert J. Schiller in seiner Studie "Mass Communication and American Empire" gut dokumentiert worden. Amerika verkauft z. B. "hardware" (Produktionsmittel) und "software" (hier: fertige Programme) wie ein fertiges Paket, gibt Hilfestellung beim Transfer von Organisationsstrukturen durch Gelehrte und Fachleute, hat bereits Ausbildungsgänge und Berufsbilder durch Imitation eigener akademischer Institutionen mit ihren Veröffentlichungen und Ausbildungsmethoden geschaffen, stellt Programme zur Verfügung, wenn die lokalen Programmgestalter ihre Sendezeit nicht ausfüllen können und trägt zum Werbeeinkommen bei, um die Organisationen zu unterstützen. So schließt sich der Kreis, indem Amerika neue Konsumbedürfnisse und Wünsche sowie jene Produkte liefert, die von der heimischen Industrie nicht oder nur in Zusammenarbeit und Unterstützung mit amerikanischen multinationalen Firmen bereitgestellt werden können. Am Ende spielt diese Art von Entwicklung durch die größeren finanziellen Gewinne den reichen Ländern alle Bälle zu. Die vergleichsweise geringen finanziellen Gewinne der Entwicklungsländer müssen durch enorme kulturelle Verluste aufgewogen werden.

\section{*}

Um den Weg durch den Wald zu erkunden, müssen wir besser definieren, was wir mit "Massenmedien" meinen. Es ist in Asien ganz offenkundig, daß wir die Massen nicht durch „Massenmedien" erreichen, außer sie sind das Beiprodukt einer mehr grundlegenden und wirkungsvolleren mündlichen Kommunikation. Ich war überrascht $\mathrm{zu}$ erfahren, wie uneffektiv "Massenmedien“ sein können, wenn es darum geht, die Massen $\mathrm{zu}$ informieren. $\mathrm{Zu}$ diesem Ergebnis kam einer meiner Studenten an der "Chinese University" in Hongkong, als er die Kommunikationsgewohnheiten und den Lebensstil eines kleinen Dorfes in der Nähe unseres Campus erforschte. Dabei liegt dieses Dorf am Fuße eines Berges, auf dessen Spitze die Rundfunkanstalt von Hongkong ihre Antennen hat. Das zur Stadt Hongkong gehörende, aber in sich geschlossene Dorf Mei Yuen New Village, hat fünf Reihen zweistöckiger ZementBlockhäuser. Darin leben ungefähr 900 Personen in etwa 88 Familien. Der Student wollte nun herausfinden, wie wirkungsvoll das Regierungsprogramm zur Familienplanung war. Er untersuchte die entsprechenden Informationen der Massenmedien. Erstens entdeckte er, daß die Dorfbewohner ein untypisches Bild abgaben und nicht in eine der drei Kategorien "traditionals, transitionals and moderns" paßten, die Daniel Lerner in seiner Arbeit "The Passing of Traditional Society" beschrieben hatte. Zweitens beobachtete er, daß das Fernsehen für Erwachsene als Informationsquelle keine größere Bedeutung hat. Niemand war des Schreibens kundig, so daß gedruckte 
Medien keinen direkten Einfluß hatten. Die Leute waren für ihre Information völlig von der mündlichen Kommunikation abhängig.

Wir im Westen haben unbewußt schon immer "Massenmedien“ mit den Massen assoziiert. In Amerika erreichen diese Medien tatsächlich die Massen, auch wenn es sich dabei um eine Einbahn-Kommunikation handelt. In Asien ist dagegen der Zugang zu den sogenannten Massenmedien weithin ein Privileg der oberen Zehntausend. K. E. Eapen vom Fachbereich Kommunikation der Bangalore Universität möchte "Massenmedien " von Kommunikation unterscheiden. Er meint, daß Rundfunk, Fernsehen und Zeitungen nur einen Bruchteil der indischen Bevölkerung erreichen. "Genau wie Reichtum sich vermehrt, so ziehen Medien andere Medien an. Wenn es in einem Haus Fernsehen gibt, hat man dort vermutlich auch Radio, Zeitungen und Magazine." Während also die Technologie den Eindruck erweckt, daß ein riesiges Land wie Indien von einem dichten Kommunikationsnetz überzogen ist, gibt es in Wirklichkeit nur Inseln der Sättigung und der Unfruchtbarkeit für die „Massenmedien" in diesem Land. Sogar in den großen Städten entdeckte Eapen viele Menschen, die zwar physisch in städtischen Gebieten wohnen, aber dennoch von den „Massenmedien" nicht berührt werden. Wenn wir unsere Aufmerksamkeit auf die Wunderinstrumente der modernen Kommunikation richten und meinen, jedermann sei darauf eingestimmt, so haben wir den kommunikativen Wert und die Wirkung etwa von Puppenspielen, Volksliedern und -tänzen sowie tausenden anderer traditioneller Kommunikationstypen in asiatischen Gesellschaften aus dem Blick verloren. Die Gefahr besteht, daß das alles verlorengeht, statt in unsere modernen Kommunikationssysteme integriert $\mathrm{zu}$ werden, die dann viel effektiver würden.

Und einen weiteren Aspekt der Ubertragung dieser „Massenmedien“-Kultur auf Asien finde ich beunruhigend. Die amerikanisch geprägte, wissenschaftliche und technische Kultur hat bestimmte erkenntnistheoretische Voraussetzungen. Der grundlegende historische Konflikt zwischen Religion und Wissenschaft scheint unmittelbar deutlich für Wissenschaftler und für Vertreter der Religion. Weitsichtige Religionswissenschaftler haben lange erkannt, daß Gott in den Händen der Wissenschaftler Schaden nimmt. Die Wissenschaft beschäftigt sich nur mit sichtbaren materiellen Phänomenen. Sie stellt Gott außerhalb ihrer Studien und Forschung, und indem sie dies tut, eliminiert sie Gott letzten Endes. Wissenschaft in diesem Sinne ist atheistisch. Mit einiger philosophischer Berechtigung behauptet eine gewisse theologische Schule heutzutage, Gott sei durch die Technologie vollständig vernichtet worden. In diesem Rahmen versuchte Harvey Cox vor einigen Jahren, Gott nach "Technopolis“ zurückzubringen, indem er nach einer Religion forschte, die Kommunikation auf derselben Ebene wie Wissenschaft und Technologie betreibt. Aber wenn man das "Spirituelle" aus der Religion verbannt, wie es Harvey Cox in "The Secular City “ tut, hat man dann nicht einen guten Grund für den Tod (oder bevorstehenden Tod) Gottes? Ich fürchte daher, daß die alten und hochentwickelten Traditionen der Religion in Asien sterben werden, wenn die dortigen Nationen diese Kultur übernehmen.

Doch Religion läßt sich in menschlichen Gesellschaften und Kulturen nicht leicht ausrotten. Und die säkulare Kultur der wissenschaftlichen, technologischen und industriellen Gesellschaft Amerikas hat ihre eigene Mythologie und ihre eigenen Götter. Es handelt sich um den Gott der Arbeit, den Gott des Fortschritts und die Göttin des Glücks. Diese Aspekte der amerikanischen Kultur, welche amerikanische "Massenmedien" nach Asien übertragen, sind weitgehend von den Fachleuten der Kommunikationsforschung übersehen worden. Tatsächlich ist die Geschichte der 
Technologie eine herausfordernde und oft vernachlässigte Geschichte. Wenn man die psychologischen Kräfte des technologischen Erbes im modernen amerikanischen Staat übergeht oder unterschätzt, so heißt das die Augen schließen vor einer Sache, die für das Verständnis der Kommunikation von höchster Bedeutung ist. Aber gerade diese Aspekte gehören zu jenem Paket, das die asiatischen Nationen annehmen wollen.

Eines muß uns klar sein. So etwas wie reine Wissenschaft gibt es nicht. Die ersten experimentell arbeitenden Wissenschaftler waren eine eigenartige Mischung von alter Mechanik und Philosophie neuen Stils. Wissenschaft und Mechanik brachten einen mächtigen Nachkommen hervor, jene Technologie, die eine besondere Fertigkeit in der Anwendung von Mechanik oder mechanischen Systemen entwickelt hat. Ein pessimistischer französischer Soziologe, Jacques Ellul, setzt in seinem Buch "The Technological Society“ Technologie mit amerikanischen "know how " gleich. Nach seiner Ansicht bedeutet Technologie all jene maschinenähnlichen Funktionen in der modernen Gesellschaft, in denen die Technik die Handlungen eines Objektes oder einer Gruppe von Objekten, einschließlich der Menschen, diktiert. Die moderne Gesellschaft erfindet diese Instrumente, und indem sie sich mechanisiert, verwandelt sie sich in eine technologische Gesellschaft mit dem Anspruch auf Effektivität. Technischer Fortschritt ist seiner Ansicht nach irreversibel und tendiert dahin, sich in einer geometrischen, nicht nur arithmetischen, Reihe auszudehnen. Alle Faktoren in dieser Gesellschaft sind letztlich den Forderungen der schnellebigen Technologie untergeordnet. Die meisten menschlichen Grundwerte werden unter dieses „know how “ eingeordnet, und Kunst und Wissenschaft sind in allen ihren Phasen der Herrschaft der Technik unterworfen. Alle Bildungsinstitutionen und Kommunikationseinrichtungen verwandeln sidh so in Instrumente der Propaganda und UUberredung. Um die Masse zu überzeugen, ist es notwendig, auch die brutalen Nebenwirkungen der Technologie anzunehmen und gleichzeitig die Ergebnisse zu ignorieren, die sie auf Kosten der Perfektion und der Lebensqualität erreicht.

Der Preis für diese technologischen Errungenschaften muß hoffentlich nicht im Sinne von Elluls düsteren Voraussagen eines Tages bezahlt werden. Ein Blick auf Japan, das wie Amerika die Technik überstrapaziert, zeigt als Resultat einen mannigfaltigen psychologischen Druck auf seine Gesellschaft und seine Institutionen. Man kann wohl sagen, daß in Nationen, die wie Amerika von der Technologie tiefgehend geprägt sind, jede Lebensphase zu einer Entwicklungsstufe der Technologie wird. Das gilt sogar für die persönlichsten Aspekte unseres Lebens. Man beachte nur die Verbreitung von populären Sexbüchern, die uns sagen, wie das Intimleben verbessert werden kann. Die meisten stellen nur eine Anleitung zur Technik dar. Es war unvermeidbar, daß auch die Kommunikationsprozesse zutiefst von der Technologie beeinflußt wurden. Kommunikatives Verhalten wandelte sich von relativ stabilen Mustern der Beziehungen in den klassischen Ausdrudksformen wie Konversation, Malerei, Tanz, Theater und Gesang zu massenhaft gefertigten und verbreiteten Materialien jeder Art. Diese neuartigen Kommunikationserzeugnisse gingen einher mit einer Verschwendung von gedrudkten, gesprochenen und fotografierten Ideen. Dies alles entwickelte sidh weder aus den psychologischen oder gesellschaftlichen Bedürfnissen der verschiedenen Schichten der amerikanischen Gesellschaft noch mit innerer und logischer historischer Notwendigkeit. Es herrschte allein das Gebot der Technologie.

Die Allgegenwart der Technologie hat viele Menschen auf verschiedene Weise umgebracht und tut es noch immer. Bevölkerungskontrolle wurde zu einem zentralen humanistischen Problem der technologischen Gesellschaft. Die Technologie scheint 
auch dieses Problem auf die eine oder andere Weise lösen zu können. Wenn Verwirrung und Entfremdung von der Natur die Lebensbedingungen dieser Art von Gesellschaft sind, dann ist dies der Preis, den eine solche Gesellschaft zahlen muß für einen schnellen Wandel im Lebensstil, den die Technologie herbeiführt. Alle Problemlösungen geraten zu einer technischen Angelegenheit: Alkoholismus, Drogensucht und Homosexualität. Auch die religiösen Menschen werden von ihrer eigenen Religion durch die Technik "geheilt". Es gibt gewisse Techniken, sich mit der Religion zu befassen, so etwa wenn man sie auf die Kirchen beschränkt und ihre Anhänger ermutigt, Bücher und Artikel zu schreiben, die aber gleichzeitig nur in ihren eigenen Kreisen gelesen werden dürfen.

Mit anderen Worten: Wir können in Asien unsere Ausbildungsprogramme für Massenkommunikation nicht fortsetzen, ohne diesen gängigen, angenommenen oder aufgezwungenen, Besitzstand zu sehen und ihn in Frage zu stellen. Jede Erfindung hat ihre Geschichte, und diese ist nicht immer so logisch verlaufen, daß sie im Nachhinein von späteren Forschern nachvollzogen werden kann. Tatsächlich sind die meisten logischen Erklärungen für Entwicklungen zur Größe der modernen Kommunikationsmuster lediglich „Geschichten“, die von den Autoren aufgrund ihrer eigenen ethischen, philosophischen und wissenschaftlichen Grundsätze von „Ursache und Wirkung" dieser Geschichte aufgepfropft wurden. Um ein besonders deutliches Beispiel zu nennen: Marshall McLuhan, dessen Werk „Understanding Media “ während meiner zehn Jahre in Asien überschwenglich von akademischen und christlichen Kreisen zitiert wurde, hielt uns davon ab, die Tricks der "Massenmedien" $\mathrm{zu}$ sehen, wie sie wirklich

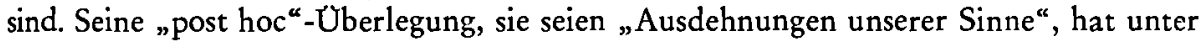
christlichen Kommunikatoren entscheidend dazu beigetragen, die Tatsache zu verschleiern, daß Massenmedien „begehrte Instrumente zur Manipulation der Masse als Wegbereitung für kapitalistische Expansion, Hegemonie einer neuen Elite im Bereich der politischen Macht und des Geldes, sowie für unzählige Tyranneien oder ,Nebenprodukte des Fortschritts' ${ }^{\prime \prime}$ sind (vgl. Gordon: The Languages of Communication). Derartige Massenmedien sind im Grunde Nebenprodukte der amerikanischen technologisch-industriellen Kultur, von der amerikanischen Gesellschaft zur ökonomischen und industriellen Expansion geschaffen und gebraucht.

$\mathrm{Zu}$ schließen, daß es unter den Erfindungen von Kommunikationsmustern keine erkennbaren, logischen Entwicklungsschemata vom Grunzen eines Höhlenmenschen bis zum Satellitenfernsehen gibt, ist weder ungenau noch entmutigend. Der ethisch denkende Historiker leidet unter derselben Frustration, weil die Geschichte der Moral ebenfalls keine klar erkennbaren, logischen Modelle aufweist. Solange wir nur auf die glitzernden technologischen Konsumprodukte im Westen sehen und die psychologischen Nebenwirkungen ignorieren, die alle Aspekte dieser amerikanisch-technologischen Umwelt durchdringen, werden wir niemals in der Lage sein, realistische Leitlinien für den künftigen Gebrauch dieser Erfindungen zusammenzustellen. Immer noch glauben wir an die vollständige Indifferenz der Technologie. Sie trägt in sich selber kein Wertsystem, keine Moralität, keine Ethik und auch keine Garantie dafür, $\mathrm{da}$ sie unsere Kommunikationsmisere mehr erleichtert als vergrößert. Um es mit dem Beispiel eines Autos zu sagen: Ohne Fahrer ist es nur eine technologische Masse. Das Auto hat Möglichkeiten, die durch den Fahrer aktiviert werden müssen. Kommunikationsinstrumente als Techniken, als gesprochene oder geschriebene Sprachen, als gedruckte "Medien“, als Film, Radio und Fernsehen betrachtet, müssen wie Autos von fähigen Leuten gefahren und kontrolliert werden, die ihre Möglidhkeiten und 
Grenzen genau kennen. Die Kommunikationsweisen, wie sie George N. Gordon etwa als erzählend, bildhaft und personifizierend versteht, sind in der Menschheit von Anfang an vorhanden. Sie sind verflochten in alle Kommunikationsvorgänge und bilden jetzt einen Teil des Inhalts der "Massenmedien“. Die Vermittlungsweisen („mediums“) und Vermittlungstechniken ("media“) sind unterschiedlich, aber bis jetzt hat unsere Überbetonung der Vermittlungstechniken dazu geführt, die Vermittlungsweisen zu vernachlässigen, und wir haben versäumt, genau festzustellen, welche Rolle sie in der Kommunikation spielen. So bin ich in zehn Jahren von einem „hardware“-Anhänger zum „software“-Mann geworden. Während meines Aufenthaltes an der Sogang-Universität änderten wir dreimal das Curriculum derart, daß wir 1973, als ich abreiste, die Schwerpunkte des Curriculums auf Theorie und Forschung verlegt und uns von der früheren Art der Ausbildung und Produktion entfernt hatten. Allerdings war ich mir bewußt: Ich setzte die Koreaner zwar hinter das Steuer, aber unser Auto war noch immer amerikanisch. Eine neuere AMIC-Ubersicht verzeichnet 136 Institutionen für Massenkommunikation in 16 asiatischen Ländern, die sich mit Lehre, Ausbildung und Forschung befassen. Soweit ich weiß, steuern sie fast alle in die falsche Richtung. Im Sommer 1976 habe ich an der "Chinese University of Hongkong" nach langen Auseinandersetzungen zusehen müssen, wie ein teures und ausgedehntes Massenkommunikations-Programm ins Leben gerufen wurde, das jede von der Natur der Sache her geforderte Kritik, wie ich sie darzustellen versuchte, ignorierte. So verließ ich Asien nach zehn Jahren mit vielen $Z$ weifeln und Enttäuschungen wegen der möglichen Verluste asiatischer Kultur und asiatischen Erbes, weil man unweigerlich und irgendwie unkritisch die amerikanisch-technologische Kultur übernimmt.

\section{*}

Wie soll es nun weitergehen? Neue Richtungen in der Ausbildung müssen überlegt werden, auch wenn sie noch nicht durchgeführt werden. Wir werden bald fähige „Massenmedien "-Praktiker im Uberfluß haben, wie es in Amerika schon lange der Fall ist. Dieser Teil der Kommunikationsausbildung braucht deswegen unser besonderes Interesse. Der neue Anstoß muß im Bereich der Forschung gegeben werden, und zwar zu einer Forschung in der Dritten Welt, zu der Asien fast ausnahmslos gehört. Gloria D. Feliciano, Direktorin des Kommunikationsinstituts der Universität der Philippinen in Manila, selbst ein Produkt des bekannten Communication Institute der Stanford-Universität mit Wilbur Schramm, ist mit Erfolg dabei, aufgrund ihrer eigenen Erfahrung in der Feldforschung aus den Grenzen jener Forschungsmethoden auszubrechen. Seit Jahren plädiert sie nahezu als einzige für Änderungen in den Forschungsmethoden. Sie stellt fest, daß methodische Fertigkeiten in der Forschung und dabei bezieht sie sich auf amerikanische Methoden - allein nicht ausreichen, sondern daß die Fähigkeit zu menschlichen Beziehungen in einer gegebenen Kultur genau so wichtig ist. Eine ausführliche umschreibende Ausdrucksweise wird in Asien der direkten und präzisen Mitteilung vorgezogen. Einfühlungsvermögen erscheint wichtiger als wissenschaftliche Rationalität. Der Forscher muß aus der amerikanischen Effektivitätsschablone ausbrechen und sich Zeit gönnen für zwischenmenschlichen Kontakt.

Und wo lägen die Schwerpunkte in der Forschung? Wir können auf unserem Weg durch den Wald nicht allein gehen. Die Bemühungen müssen interdisziplinär scin. Schon zu lange haben wir uns auf unsere kleinen, privaten Räume in Studium und Forschung beschränkt. Wir brauchen z. B. die Hilfe von Entwicklungs-Anthropologen, die sich mit interkultureller Kommunikation und angewandter Anthropologie befaßt 
haben. Akkulturations-Studien konzentrieren sich darauf festzustellen, wie Verhalten, Werte und Symbole - um nur einige Aspekte zu nennen - von einer dominierenden ausländischen Kultur übernommen werden. Die angewandte Anthropologie ist andererseits interessiert an Kommunikation als einem Weg für die Einführung einer planmäßigen kulturellen Veränderung.

Beispiel einer solchen Forschung könnte der Gebrauch der Symbolik sein. Bücher und literarische Texte, die im Lauf der Geschichte den tiefsten Eindruck auf die Menschheit machten, gebrauchten die Form der Parabel und oft auch Verse. Die poetische Parabel fordert, als komplexe Form des Symbolischen, vom Leser keine unmittelbare individuelle Beziehung zu einer bestimmten Erfahrungswelt. Stattdessen behandelt sie vieldeutige Stoffe, die vom Leser und Hörer zu jeder Zeit und in jeder Umgebung angewendet werden können. Die meisten philosophischen Allegorien und Dramen bedienen sich dieser Art vieldeutiger Kommunikation, wie es übrigens auch die heiligen Bücher aller bekannten Religionen tun. Es ist hier nicht der Ort zu einem längeren Diskurs über das Symbol, doch seien noch einige Bemerkungen erlaubt. Das Symbol ist eine Idee, die in ein scheinbar beziehungsloses Surrogat ihrer selbst verwandelt wurde, welches für die ursprüngliche Idee steht. Es ersetzt die unmittelbare Erfahrung. Dieses Symbol gibt dem Einzelnen die Möglichkeit, seine Erfahrung über die unmittelbare Umgebung hinaus auszuweiten. Es räumt ihm die Möglichkeit ein, etwas stellvertretend zu erfahren, was vielleicht physisch unmöglich, zu teuer, zu bedrohlich, zu schwierig oder unbequem. ist.

Nun ist klar, daß Gesellschaften, Kulturen und Völker sich nicht statisch verhalten. Dies gilt auch für Symbole. Der Symbolgehalt im Leben einer Kultur tendiert dahin, sich auszuweiten und zu verändern, und bedarf daher ständiger Beschneidung. Dies wird deutlich, wenn man etwa die uns am ehesten vertraute Symbolstruktur, deren wir uns bedienen, die Sprache betradten. Wörter und Redensarten ändern Form und Gehalt mit dem Wandel in Kultur und Gesellschaft. Es gibt ein ständiges Bedürfnis nach neuen Symbolen, die ältere ersetzen und aus dem Kommunikationsfluß abdrängen. Doch muß hier ein Gleichgewicht zwischen Änderung und Stabilität erhalten bleiben.

In unseren zunehmend komplexen Gesellschaften von heute mit ihren zahlreichen Subkulturen, in denen die "Massenmedien“ ihren Einfluß ausüben, gibt es viele Erscheinungsweisen symbolischer Worte und Handlungen, die bislang nur zum Teil von Soziologen, Dramatikern und Schriftstellern erforscht worden sind. Um diese Strömungen wirklich zu verstehen, muß ein Forscher wohl in einer solchen Subkultur leben, an ihrer symbolischen Struktur teilhaben und als Beobachter gleichzeitig eine gewisse Distanz zu ihr halten. Letzteres ist für den einheimischen wie auch für den "ausländischen“ Beobachter schwer. Der eine hat durch Erziehung und Ausbildung eine zu großc Nähe, der andere durch Sprache und Erfahrung vielleicht zuviel Distanz. Hier könnten gemeinsame Bemühungen am besten zum Ziele führen. Meine siebzehnjährige Abwesenheit von der Heimat hat mir $z$. B. die notwendige Distanz verschafft, Amerika mit neuen Augen zu sehen.

Unter sozialem Aspekt können Symbole die Lebensform (the how) der Gegenwart mit dem Lebensgehalt (the what) der Vergangenheit verbinden. Im Prozeß der Stabilisierung unserer Erfahrungen und unserer Kultur geben uns Symbole die Verbindung von Vergangenheit, Gegenwart und Zukunft zu einem Ganzen, um in unserer Erïahrungswelt jene Konturen zu zeigen, auf die hin unser künftiges Leben aus- 
gerichtet und gelebt werden kann. In einer pluralistischen Gesellschaft und Welt, die uns von den "Massenmedien“ direkt oder indirekt vorgestellt wird, gibt es eine gewaltige Verwirrung im Symboldenken und vermutlich auch eine kulturelle Inkompatibilität von universalen Symbolen, die alle Bereiche menschlicher Erfahrung und Existenz betreffen. Weil wir in einem „Weltdorf " ("global village") leben, um eine von McLuhans Analogien zu gebrauchen, müssen wir lernen, miteinander in Kommunikation zu treten, ob wir wollen oder nicht. Deswegen ist es unsere Aufgabe, herauszufinden, wie kulturelle Gräben zu überbrücken sind, und allgemeine Symbole zu finden, soweit dies überhaupt möglich ist.

Gesellschaftskritiker haben sich laut gegen eine Trivialisierung der Kultur gewandt, die durch die "Massenmedien" praktiziert werde. Wenn eine solche Trivialisierung tatsächlich geschieht, dann stellt sie tatsächlich einen Angriff auf unsere Symbolsysteme dar. Kritiker bemängeln, daß drei Institutionen unserer Zeit, nämlich die Werbeindustrie, einschließlich Fernsehen und Film, die Ausbildung auf allen Ebenen, vom Kindergarten bis zur Universität, und die Bürokratie fehlgesteuert und tief in die Trivialisierung des symbolischen Prozesses verwickelt sind. Die Werbeindustrie, so argumentieren diese Kritiker, habe uns konsumorientiert, materialistisch und passiv werden lassen. Unsere Bildungseinrichtungen auf den unteren Ebenen zwingen die Kinder eher dazu, die symbolische Struktur zu übernehmen als auf die Gestaltung der Symbolwelt Einfluß zu nehmen, und auf den höheren Ebenen sind Colleges und Universitäten zu Diplomfabriken geworden. Sie produzieren fähige Techniker, Ingenieure, Ärzte und Rechtsanwälte für den Abschmierdienst an den symbolischen Rädern der Kultur. Schließlich hat die Bürokratie den komplexen Symbolismus einer Autorität geschaffen, die die Großunternehmungen, Stiftungen, große Ausbildungsstätten und Regierungen erhält, schützt und verewigt. Sie ist der unpersönliche Schiedsrichter über das menschliche Schicksal in einer Massengesellschaft. In Amerika gibt es für die üblen Wirkungen dieser unglücklichen Situation anschauliches Material in den Watergate- und Lockheed-Skandalen.

Und was wird aus dem Forscher und Theoretiker, wenn er sich dieser neuen Ausbildung unterzogen hat? Ich würde ihn einen radikalen Humanisten nennen. Ein solcher Mensch fragt nach den Prämissen der Gesellschaft und fürchtet sich nicht, Ideen und Lösungen aufzugreifen, die absurd klingen mögen; er befreit sich selbst von götzendienerischem Denken; ständig erweitert er sein Bewußtsein und sieht phantasievoll und schöpferisch neue Möglichkeiten und Ansprüche voraus. In den siebziger Jahren, nach der Invasion in Kambodscha, betrachtete Arnold Toynbee Amerika mit großer Besorgnis: „Vielen Europäern erscheint, so glaube ich, Amerika im Augenblick als das gefährlichste Land der Welt, weil es zweifellos am mächtigsten ist." Ich möchte nicht, daß die Asiaten Amerika und seiner "Massenmedien"-Technologie mit Angst begegnen. Ich möchte nur, daß sie diese und ihre eigene Kultur in radikal humanistischer Perspektive sehen, damit sie einer Verschmelzung von beiden, wenn sie möglich ist, zur Integration und Vitalität, nicht aber zu Vorherrschaft und Untergang geführt werden.

\section{Bibliographie:}

1. Eapen, K. E.: Communication and Behavioural Change. 125 U. V. C. E., K. R. Circle, Bangalore 560 001, India.

2. Ellul, Jacques: The Technological Society. New York 1965. 
3. Feliciano, Gloria D.: An Overview of Communication Research in Asia: Status, Problems and Needs. In: "Papers of the East-West Communications Institute“, No. 6, Honolulu, Hawaii 1973; Feliciano and Benjamin V. Lozare: Doing Research in Rural Asia: Problem of Methods. In: „Media Asia“, 1:1, 1974, pp. 15-20.

4. Foster, George M.: Tradition Societies and Technological Change. New York ${ }^{2} 1973$.

5. Goodenough, Ward Hunt: Cooperation in Change. New York 1963.

6. Gordon, George N.: The Languages of Communication. New York 1969.

7. Gordon: Communication and Media. New York 1975.

8. Hamelink, Cees: Perspectives for Public Communication. Baarn 1975.

9. Langer, Susanne K.: Feeling and Form. New York 1953.

10. Langer: Philosophy in a New Key. New York 1948.

11. Lerner, Daniel: The Passing of Traditional Society. Glencoe 1958.

12. Lerner and Schramm, Wilbur: Communication and Change in the Developing Countries. Honolulu 1967.

13. Ruesch, Jürgen: Clinical Science and Communication Theory. In: Matson, Floyd W. and Ashley Montagu (Ed.): The Human Dialogue. New York 1967, pp. 51-70.

14. McLuhan, Marshall: Understanding Media. New York 1964.

15. Schiller, Herbert I.: Mass Communications and American Empire. New York 1969.

16. Schramm, Wilbur: Mass Media and National Development. Stanford 1964.

1\%. Whitehead, Alfred North: Symbolism. New York 1958.

18. Hamelink, Cees (Ed.): Communication Research in the Third World, Lutheran World Federation, Dept. of Studies. Geneva 1976.

19. UNESCO: Training for Mass Communication, UNESCO's Office of Publications, No. 73, 1975.

\section{S U M M A R Y}

The author critically views the role hardware still plays in the communications-training in Asia. He sees this in relation with the American presence within the academic development of Asian countries, where American technology very often leads to a mere economic concept of development. Thus the treasures of Asian culture are lost and the information-gaps between the Third World and the West are widened. For communications training this leads to some consequences: The training in the technical field of the "Mass Media“, which very of ten don't reach the people, has to be substituted by methods of interpersonal communication and the study of anthropology. Here traditional means like songs, dance, theater are to be considered. Of some importance along these lines is the research in symbols which are open to intercultural communication. The symbol can also interlink past, present and future in history.

\section{RESUME}

L'auteur analyse tout d'abord de façon critique le rôle que joue le hardware dans les branches de la communication des universités asiatiques jusqu'd nos jours. Il présente la présence des américains dans l'entreprise scientifique de ce pays, lattrait de la culture americano-technologique, de même que la restriction de la notion de développement à un développement purement économique comme causes de ce développement. Les causes en sont des pertes culturelles irremplaçables en Asie et une forte chute de l'information, du monde occidental jusqu'au tiers monde. 
L'auteur tire de cette analyse d'importantes conséquences pour l'entraînement à la communication. La formation à la maîtrise technologique des "mass media " (qui, selon Mitchell, n'atteignent pas les masses asiatiques) devrait être complétée par une introduction à des méthodes personnelles et par une recherche antropologique et interdisciplinaire. Pour cela, on devrait mettre à nouveau fortement l'accent sur les exemples asiatiques traditionnels de communica. tion, tels le chant, la danse et le théatre. A ce propos, l'auteur attribue un rôle important à l'exploitation de ces symboles, puisque cela représente une forme de communication ayant fait ses preuves à maintes reprises, forme qui ne repose pas sur une base d'expérience concrête et qui est ouverte à une recherche et 'une communication interculturelle. Par là, le symbole peut faire le lien entre l'histoire, le présent et le futur.

\section{RESUMEN}

El autor se enfrenta primero críticamente con el papel que desempeñaron hasta hoy los hombres de Hardwar en el campo de la comunicación social en las universidades asiáticas. Como causas de este desarrollo cita la presencia de americanos en los centros científicos de esos países, el atractivo de la cultura tecnológioa americana y la limitación del concepto de desarrollo al desarrollo meramente económico. Las consecuencias son una irreparable pérdida cultural en Asia y una fuerte corriente de información del mundo occidental al Tercer Mundo. De este importante análisis saca el autor consecuencias para el ejercicio de la comunicación. La formación para el dominio tecnológico de los »medios de difusión « (que según Mitchell aún no alcanzaron los asiáticos), debería completarse con la introducción de métodos personales y mediante investigación interdisciplinar antropológico-cultural. Deberían acentuarse de nuevo muestras de comunicación tradicionalmente asiáticas, tales como el canto, la danza y el teatro. El autor concede un papel importante en este contexto a la investigación de los símbolos, que representan un modo de comunicación que se mostró reiteradamente eficaz pues no se remite a un fondo de experiencias concretas y por ello queda abierto para la comunicación y la investigación intercultural. El símbolo puede además servir de enlace entre historia, presente y futuro. 